\title{
CT Colonography (virtual colonoscopy)
}

\author{
PIERRE VASSALLO
}

hin-section multi-detector row
computed tomographic (CT) colonographyis a powerful tool for the detection and classification of colonic lesions.

Isotropic imaging (ie equal voxel size in all three planes) of the colon with thin collimation has become standard and provides high-quality multi-planar reformatted (MPR) images and threedimensional (3D) assessment of the entire colon, while allowing excellent visualisation of all other intra-abdominal organs.

A relatively clean, dry, and welldistended colon can be achieved with careful patient preparation, thereby avoiding the problem of residual stool and fluid. Patients generally undergo cathartic cleansing with oral administration of laxatives. Better results are obtained if patients also follow a low-fiber diet for 2-3 days before the examination and a clear liquid diet on the day of the examination. We use a combination of both techniques.
CT colonography is particularly useful as an immediate follow-up after failed optical colonoscopy, as the patient's bowel is already prepped. Failed optical colonoscopies may be due to a long and tortuous colon (eg sigma elongatum), bowel kinking (eg due to adhesions) and due to bowel obstruction (egan obstructing colonic cancer). CT colonography is able to view the colon beyond these physical impediments.

Bowel distention is performed with the patient in the left decubitus or supine position. A thin, flexible rubber catheter (eg, a small-gauge Foley catheter) is placed in the rectum, followed by gentle insufflation (either automated or manual) of carbon dioxide $\left(\mathrm{CO}_{2}\right)$ or air to patient. Tagging of faecal material can be achieved with use of barium or iodinated contrast material given orally prior to scanning.

Thin-section CT is ideally performed with a multi-detector row CT scanner with the patient first in the supine and then in the prone position. An initial scout view obtained prior to scanning in each position helps ensure adequate distention of the colonic segments, with additional $\mathrm{CO}_{2}$ or air being insufflated if required. Colonic lesions can be enhanced with the intravenous injection of iodinated contrast agent; this allows better delineation of the lesion from adjacent normal bowel and better visualisation of invasion of the mesentry and adjacent structures.

The full abdomen is imaged in one breath-hold (7-13 secs) with a 64-Slice CT scanner and image slice thicknesses down to $0.6 \mathrm{~mm}$ can be generated allowing for artifactfree multiplanar (axial, sagittal and coronal) images of identical high resolution (Fig $1 \mathrm{a}, \mathrm{b}, \mathrm{c}$ ) and also 3D endoluminalimaging (Fig 1d).A flythrough 3D CT colonography can best appreciated on a video clip (Fig 1e). Simultaneously available 2D and 3D image displays allow rapid correlation for the evaluation of any suspected finding. A further display modality is the colonographic view, which is
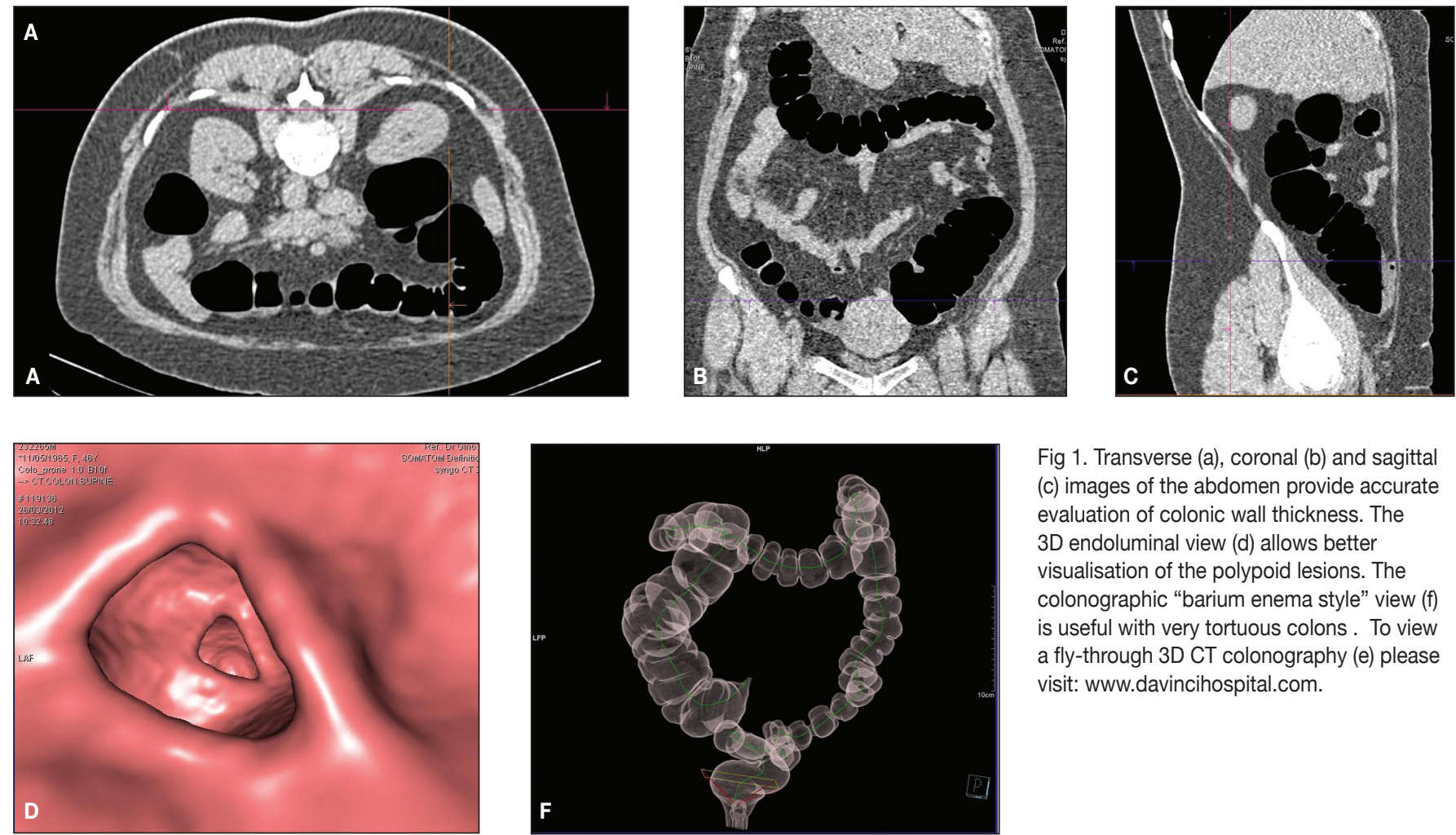

Fig 1. Transverse (a), coronal (b) and sagittal (c) images of the abdomen provide accurate evaluation of colonic wall thickness. The $3 \mathrm{D}$ endoluminal view (d) allows better visualisation of the polypoid lesions. The colonographic "barium enema style" view (f) is useful with very tortuous colons. To view a fly-through 3D CT colonography (e) please visit: www.davincihospital.com. 

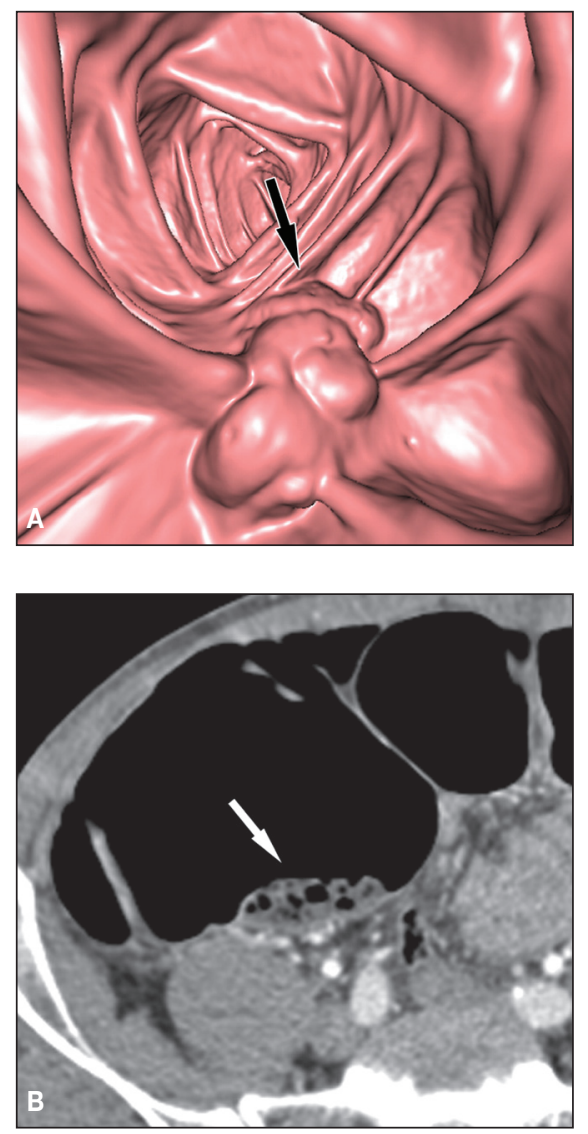

Fig 2. Residual stool appears as a broadbased, polypoid filling defect (arrow) on the 3D endoluminal image (a). The transverse image (b) no contrast enhancement, inhomogeneous texture and trapped gas in this pseudolesion (arrow).

reminescent of old barium enema examinations, however it does not suffer from the problems of overlapping bowel loops or kinks as it is a rotatable 3D image (Fig 1f).

The most common artifact that may cause some difficulty on CT colongraphy is the presence of stool.

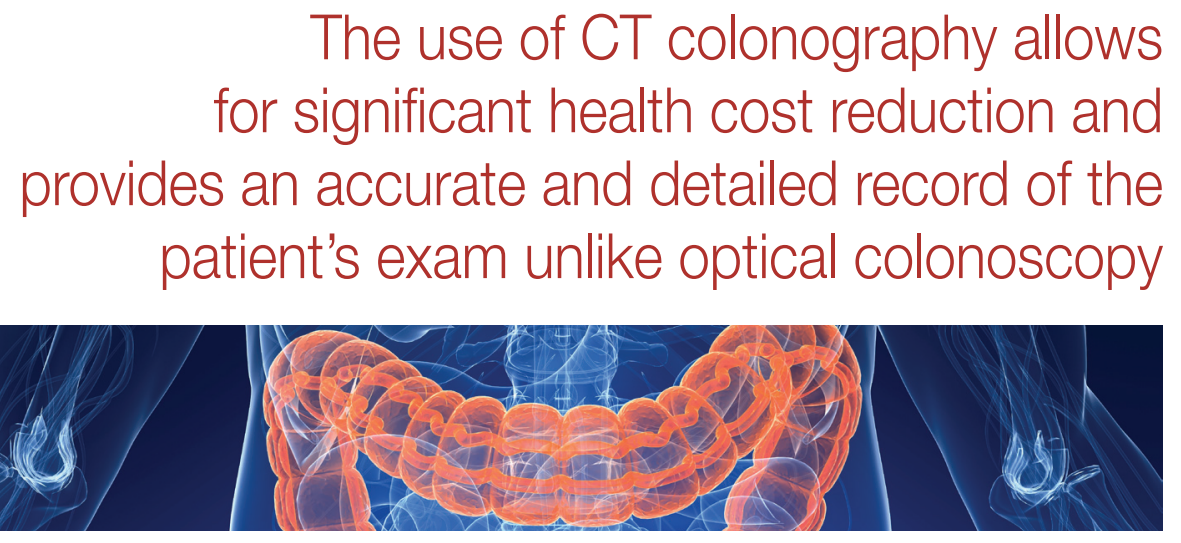

Here simultaneous evaluation of 3D and multiplanar thin slice images can resolve the problem (Fig 2) as faeces tends to contain gas bubbles and show inhomogeneous structure. Also faecal material will not enhance with intravenously administered contrast material, but will do so with orally administered contrast material.

Colonoic spasm may be confused with a stenotic lesion, however evaluation of both prone and supine views in both 2D and 3D modalities will clarify the issue (Fig 3)

Residual fluid and inadequate gaseous distension of the colon may obscure polypoid lesion, however scanning in both the supine and prone positions results in movement of both fluid and gas due to gravity; those lesions obscured on the prone scan are exposed on the suprine scan (Fig 4).

Both polypoid and invasive colonic lesions are well visualised with CT colonography (Fig 5). CT colonography / Virtual Colonoscopy has been shown to have a sensitivity of $90-96 \%$ for polypoid lesions down to $6 \mathrm{~mm}$ in diameter and a negative predictive value of $96 \%$ through numerous publications on the subject over the last 5 yrs when compared with optical colonoscopy. Virtual colonoscopy is now listed by the $\mathrm{NCl}$ (National Cancer Institute, of the $\mathrm{NIH}$ ) as one of trecommended methods for colonic cancer screening.

Diverticula may be confused with polyps when 3D navigation is used for primary data evaluation. On 3D virtual endoscopic images, the diverticular orifice appears as a complete dark ring when seen en face, whereas a polyp shows incomplete ring shadowing (Fig 6).

CT colonography allows for distinction of intrinsic bowel wall lesions from extrinsic lesions impinging on the colon unlike optical colonoscopy or barium enema examinations. The latter examiniations only visualise mucosal contours of the colon, whereas CT colonography can assess wall thickness and all extracolonic organs (Fig 7).
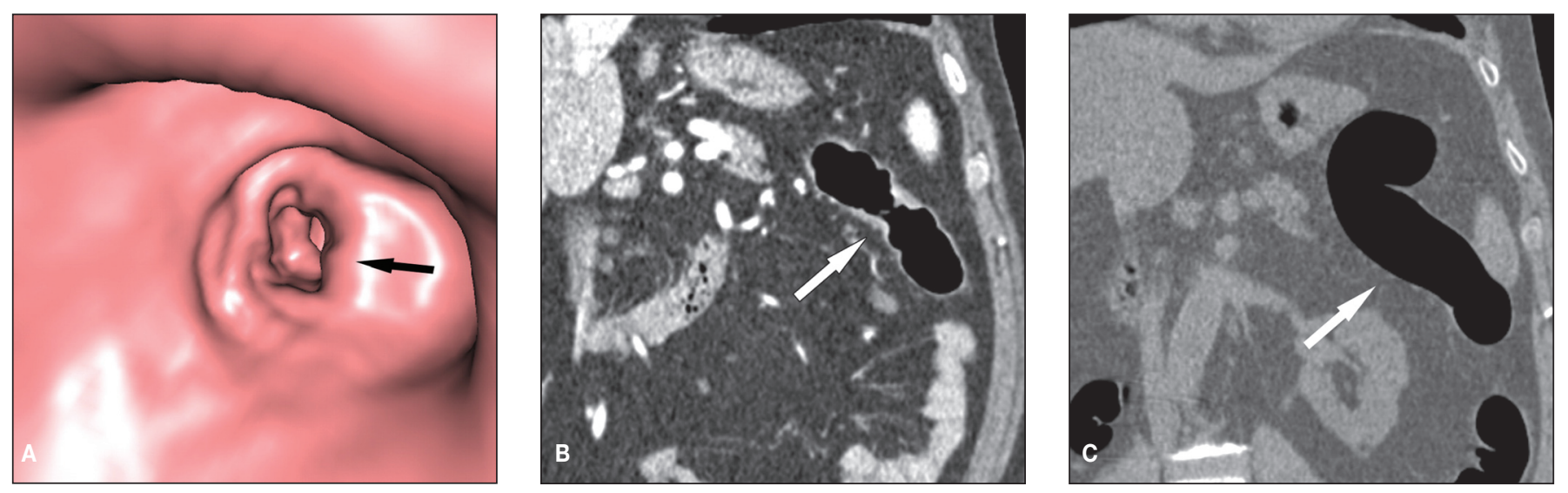

Fig 3. Segmental colonic spasm in the descending colon shows an irregular, circular narrowing of the colonic lumen (arrow) on the 3D endoluminal image (a) that simulates a stenosis. The transverse scan (b) shows focal, irregular circular wall thickening (arrow) with IV contrast enhancement. The prone scan (c) shows a normal smooth colonic wall without signs of stenosis or wall thickening (arrow) that indicate that the spasm has relaxed. 

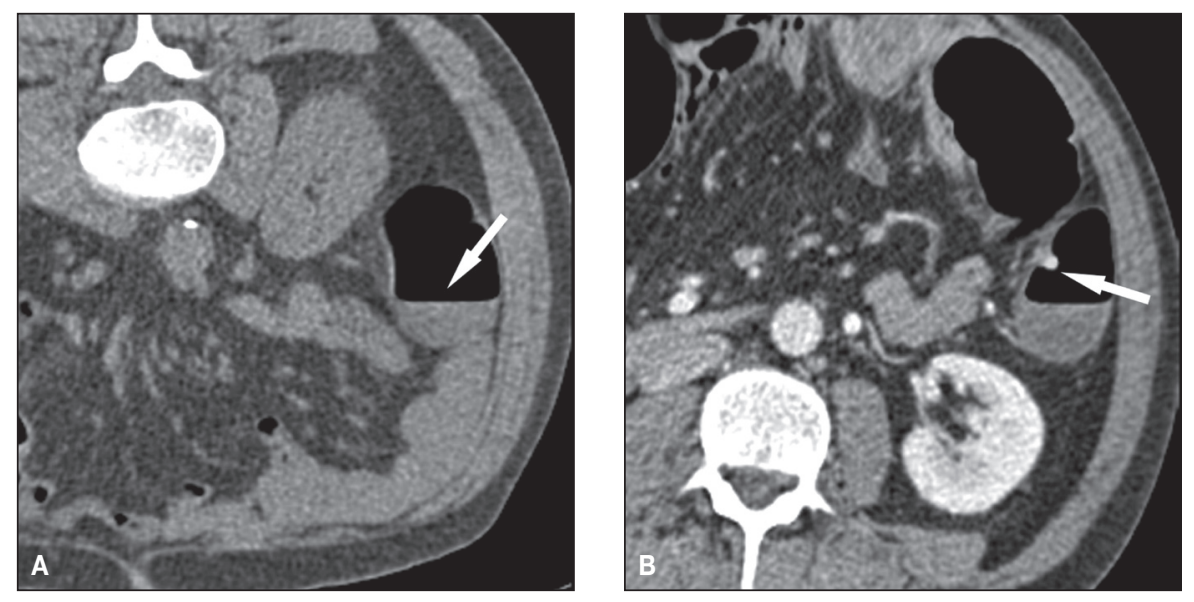

Fig 4.Fluid (arrow) obscures a descending colonic polyp in the supine position (a), but the polyp (arrow) is well seen in the prone position (b).
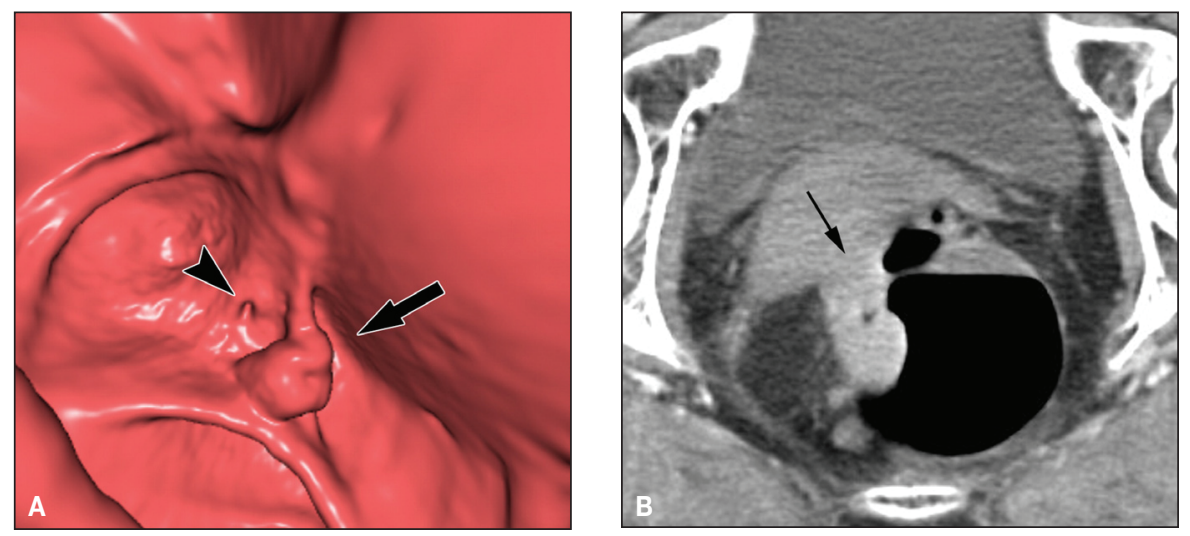

Fig 5. Sessile polypseen in the caecum (arrow) with adjacent appendiceal orifice (arrowhead) (a). Invasive rectal cancer (arrow) on 2D transverse image (b).
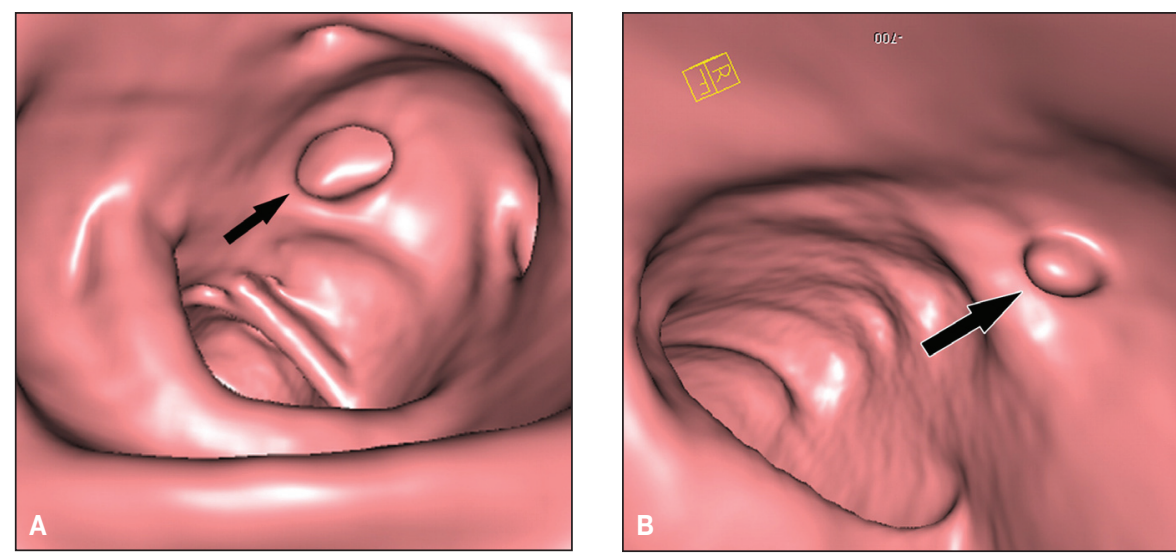

Fig 6. Full black ring seen with a diverticulum (a) but incomplete ring seen with a polyp (b) on 3D image.
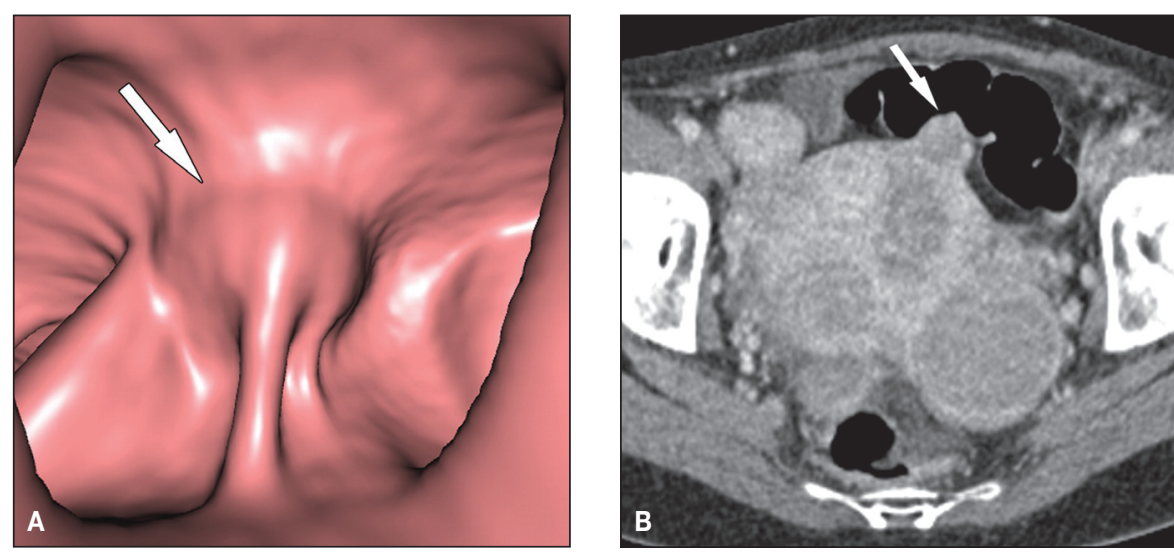

Fig 7.Extrinsic compression (arrow) noted on 3D image (a) confirmed to be due to a uterine fibroid (arrow) on the 2D image (b).
The only significant potential complication of CT colonography is bowel perforation (Fig 8). This is almost always related to the presence of inflammatory bowel disease or a constricting colonic lesion, but can also occur after snaring of colonic polyps. CT allows early detection of this complication unlike optical colonography. The likelihood of a performation with CT colonography is low $(0.03 \%)$ particularly when compared to optical colonography $(0.13 \%)$. Patient admission and observation are required in case of perforation. Surgerical intervention is rarely needed. After snare biopsies, CT colonography should be postponed by 4 weeks.

CT colonography can largely replace barium enema examinations. Optical colonoscopy can also be replaced by CT colonography, but not in the case of symptomatic patients where the requirement for biopsy is more likely. The use of CT colonography allows for significant health cost reduction and provides an accurate and detailed record of the patient's exam unlike optical colonoscopy, which provides a few photographic images at best. Availability of the full 3D data set for review and eventually for comparison with future exams is only possible with CT colonography. $\mathbb{S}$

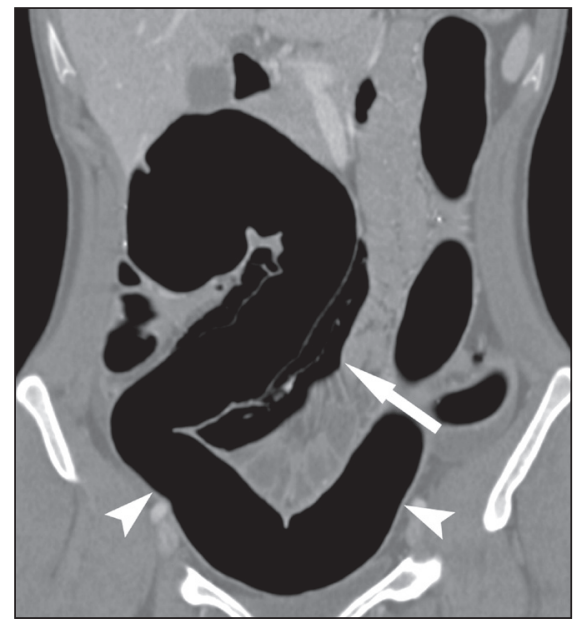

Fig 8. Colonic perforation seen on supine coronal CT scan shows pericolic air (arrow) around the transverse colon related to the perforation. Flattening and disappearance of the haustra is also seen (arrowheads) due to inflammatory bowel disease. 